\title{
La utilidad de los REA y su impacto en el aprendizaje de educación superior
}

\section{Amparo Bonet Juan ${ }^{a}$, Antonio Giménez-Morera ${ }^{\mathrm{b}}$ y Jordi Capó Vicedo}

a Universitat Politècnica de València, Department of Economy and Social Sciences, Camino de Vera s/n, 46022, Valencia, Spain, ambojua@doctor.upv.es, bUniversitat Politècnica de València, Department of Economy and Social Sciences, Camino de Vera s/n, 46022, Valencia, Spain angimo1@doctor.upv.es, c Centre for Research in Business Management (CEGEA), Universitat Politècnica de València, Spain. jorcavi0@esp.upv.es.

\begin{abstract}
Open educational resources (OER) are a key in educational innovation and collaborative learning. Consequently, the opportunity to collaborate provides facilitates the exchange of knowledge. This paper represents the experience of using Open Educational Resources (OER) in higher education and the impact. The study concludes with a proposal for collaborative learning through an OER for the logistics subject in international trade of higher education.
\end{abstract}

Keywords: open educational resources, educational innovation, collaborative learningy, higher education.

\begin{abstract}
Resumen
Los recursos educativos abiertos (REA) han sido clave en la innovación educativa y en el aprendizaje colaborativo. En consecuencia, la oportunidad de colaborar y compartir que proporcionan, facilitan el intercambio de conocimientos, desarrollo de capacidades y acceso universal al aprendizaje $y$ enseñanza de calidad. El presente artículo representa la experiencia de uso de Recursos Educativos Abiertos (REA) en educación superior y se analiza el impacto en su aprendizaje. El estudio concluye con una propuesta curricular para el aprendizaje colaborativo a través de un REA para la asignatura de logística en el ciclo superior de comercio internacional.
\end{abstract}

Palabras clave: recursos educativos abiertos, innovación educativa, aprendizaje colaborativo, educación superior.

\section{Introducción}

Cuando se habla de innovación educativa, uno de los aspectos importantes es el correcto uso de las tecnologías de la información y la comunicación (TIC) en que sucederá el aprendizaje de un concepto, una noción o una idea. La oportunidad estratégica para mejorar el proceso de aprendizaje viene de la mano de los Recursos Educativos Abiertos (REA).

Los REA son definidos por la UNESCO (en Foro de 2002 sobre las Incidencias de los Programas Educativos Informáticos Abiertos (Open Courseware) y posteriormente en 
la Declaración de París de 2012 sobre los REA) como: "Materiales de enseñanza, formativos o de investigación en cualquier soporte, digital o de cualquier otro tipo, que sean de dominio público o que hayan sido publicados bajo una licencia abierta que permita el acceso gratuito, así como el uso, modificación y redistribución por otros sin ninguna restricción o con restricciones limitadas."

Algunos autores afirman que, no existe una única manera de enseñar, como tampoco hay una única manera de aprender. Sin embargo, cada forma de enseñar genera aprendizajes distintos. Es por ello que, estos recursos educativos abiertos abren nuevas posibilidades a la innovación de la estructura educativa tradicional, permitiendo tanto a estudiantes como a docentes disponer de recursos interactivos, dinámicos, flexibles y totalmente libres, capaces de facilitar el acceso a un aprendizaje distinto y de calidad.

En los últimos años, el auge de los REA ha sido clave en la innovación educativa y en el aprendizaje colaborativo. En consecuencia, la oportunidad de colaborar y compartir que proporcionan, facilitan el intercambio de conocimientos, desarrollo de capacidades y acceso universal a aprendizaje y enseñanza de calidad.

Por todo ello, a continuación se presenta la programación didáctica del módulo de logística perteneciente al Ciclo Superior de Comercio Internacional para el que se ha elaborado un REA, de utilidad tanto en el ciclo presencial como semipresencial.

\section{Antecedentes}

Resulta de interés conocer las posibilidades de generar recursos educativos abiertos y alternativas de aprendizaje afines con estas nuevas tendencias actuales en la Educación Superior, tal y como se establece en el informe Horizon Report 2015.

El presente trabajo tiene como objetivo compartir la experiencia de uso de Recursos Educativos Abiertos (REA) en educación superior y su impacto en su aprendizaje de la asignatura de logística mediante la propuesta curricular de la misma con la metodología de REA. El REA incluye:

1. Contenidos de aprendizaje: materiales para el curso, módulos, contenidos, objetos de aprendizaje, etc. istepi

2. Herramientas: Software para la creación, entrega, uso y mejora del contenido de aprendizaje abierto, incluyendo búsqueda y organización de contenido, sistemas de gestión del aprendizaje (LMS), herramientas de desarrollo de contenidos, y comunidades de aprendizaje en línea. ischp:

3. Recursos de implementación: Licencias de derechos de autor que promuevan la publicación abierta de materiales, principios de diseño y adaptación local de

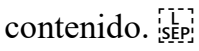

\subsection{Datos generales}

La siguiente tabla, presenta en los datos descriptivos del REA que lleva por título "Técnica de almacén". 
Tabla 1. Datos descriptivos del REA

\begin{tabular}{|c|c|}
\hline Título & “Técnica de almacén” \\
\hline Tema & La logística en el Comercio Internacional \\
\hline Descripción & $\begin{array}{l}\text { La finalidad del mismo es alcanzar la competencia general del título, } \\
\text { consistente en desarrollar actividades de distribución y comercialización de } \\
\text { bienes y/o servicios, y en gestionar un pequeño establecimiento comercial, } \\
\text { aplicando las normas de calidad y seguridad establecidas y respetando la } \\
\text { legislación vigente. }\end{array}$ \\
\hline Edad & 18 años de edad en adelante \\
\hline Número de alumnos & 25 alumnos aprox. \\
\hline Etapa & $\begin{array}{l}\text { Formación Profesional superior. El proyecto se desarrollará principalmente } \\
\text { con los estudiantes de } 1^{\circ} \text { de Grado Superior del Ciclo de Comercio } \\
\text { Internacional. Sin embargo, aunque el recurso vaya dirigido principalmente a } \\
\text { módulos de la familia de Comercio, también puede utilizarse para módulos } \\
\text { de la familia de Administración. }\end{array}$ \\
\hline Áreas educativas & - $\quad$ T.S. Comercio Internacional (LOE) \\
\hline Módulos & - $\quad$ Logística (1GS Comercio Internacional) \\
\hline Duración estimada & Trimestral \\
\hline Competencias básicas & $\begin{array}{l}\text { Idiomas, digitales, aprender a aprender, sociales y cívicas, iniciativa y } \\
\text { emprendimiento }\end{array}$ \\
\hline
\end{tabular}

Fuente: Elaboración propia

\subsection{Objetivos}

1. Organizar el espacio físico y el funcionamiento del almacén, teniendo en cuenta la eficiencia en el sistema de distribución interna y la normativa de seguridad e higiene.

2. Gestionar las existencias en el almacén de acuerdo con los criterios establecidos, asegurando el nivel de servicio al cliente y la optimización del coste de los "stocks".

3. Organizar el proceso de distribución internacional de los pedidos, de manera que la entrega se efectúe en los plazos establecidos.

4. Gestionar y controlar el envase y embalaje de las mercancías para asegurar su perfecto estado durante el transporte internacional.

\subsection{Resultados de aprendizaje y criterios de evaluación}

1. Planifica las tareas diarias necesarias para realizar las operaciones y flujos de mercancías del almacén, optimizando los recursos disponibles. 
Criterios de evaluación:

a) Se ha organizado la información de las operaciones y flujos previstos en el almacén durante un determinado periodo de tiempo.

b) Se han asignado las tareas diarias del almacén al personal, de acuerdo con los tiempos asignados a cada actividad.

c) Se han transmitido correctamente las órdenes y/o instrucciones de trabajo al personal correspondiente.

d) Se han registrado las entradas y salidas de mercancías previstas del almacén en el sistema de gestión de stocks.

e) Se ha realizado el control diario del stock del almacén, garantizando las necesidades de los clientes internos o externos.

f) Se han utilizado medios de comunicación y transmisión electrónicos (EDI) para la coordinación y seguimiento de mercancías en el almacén.

2. Aplica técnicas de recepción y disposición de mercancías en el almacén, controlando los tiempos de manipulación y las condiciones de almacenamiento en función de las características de la mercancía.

Criterios de evaluación:

a) Se ha organizado la descarga de las mercancías de los vehículos, asignándole el muelle correspondiente del almacén.

b) Se ha verificado la correspondencia entre la mercancía descargada y la documentación que la acompaña.

c) Se han investigado las causas por las cuales se ha devuelto mercancía al almacén.

d) Se han identificado las instrucciones de descarga y manipulación que contienen las fichas de cada mercancía, de forma que se asegure su integridad y trazabilidad.

e) Se han calculado los tiempos de manipulación de las mercancías, optimizando las operaciones y cumpliendo con la normativa de seguridad y prevención de riesgos laborales. f) Se ha organizado la desconsolidación de la carga y el desembale de las mercancías.

g) Se ha asignado la ubicación adecuada a la mercancía recepcionada, teniendo en cuenta sus características de caducidad y rotación, entre otras.

3. Determina las condiciones de preparación de pedidos y la expedición de las mercancías del almacén, optimizando tiempos y recursos de acuerdo con la normativa vigente en materia de manipulación y transporte.

Criterios de evaluación:

a) Se han transmitido las instrucciones de preparación de pedidos por el sistema de gestión implantado. 
b) Se ha optimizado la colocación de mercancías en las unidades de carga, minimizando huecos y asegurando la estiba de las mismas.

c) Se ha determinado el método de preparación de pedidos y/o embalaje más adecuado a las características de la mercancía y del almacén.

d) Se ha diseñado el sistema de abastecimiento de mercancías a la zona de preparación de pedidos, optimizando las operaciones necesarias.

e) Se ha realizado la señalización, rotulación, codificación y etiquetado, entre otras tareas, de las mercancías que van a ser expedidas.

f) Se ha confeccionado la documentación que acompaña a la mercancía expedida.

g) Se han considerando las condiciones del seguro más favorables para la empresa, en el transporte de la mercancía expedida.

h) Se ha elegido el medio transporte más adecuado para la mercancía expedida.

4. Aplica técnicas de gestión de stocks de mercancías en el almacén, analizando parámetros de control y realizando inventarios.

Criterios de evaluación:

a) Se han calculado los parámetros de gestión y control de inventarios utilizados habitualmente en el almacén.

b) Se han establecido criterios de cuantificación de las variables que detecten las desviaciones y roturas de stock.

c) Se han establecido sistemas de control de inventarios realizados por medios convencionales o informáticos.

d) Se han establecido normas o protocolos de funcionamiento de almacén que regularicen las diferencias encontradas, en su caso, en la realización de inventarios.

e) Se han identificado las causas por las que pueden existir descuadres entre el stock físico y el contable.

f) Se han propuesto medidas que corrijan los descuadres de almacén, transmitiendo las incidencias de acuerdo con las especificaciones establecidas.

5. Programa las actividades diarias del personal del almacén, garantizando el desarrollo efectivo y eficaz de las actividades del almacén.

Criterios de evaluación:

a) Se ha determinado el número de trabajadores asignado a cada operación de almacén en función de las órdenes y especificaciones recibidas.

b) Se han asignado trabajadores a cada tarea del almacén, respetando la normativa laboral y de seguridad y prevención de riesgos laborales. 
c) Se han establecido sistemas de comunicación de las instrucciones de las órdenes y actividades diarias que tiene que realizar el personal del almacén.

d) Se han establecido métodos de control de desarrollo de órdenes y actividades del personal de almacén, de acuerdo con los sistemas de calidad implantados por la organización.

e) Se han establecido cronogramas para realizar las operaciones habituales del almacén que mejoren la productividad.

f) Se han caracterizado las necesidades de información y formación del personal del almacén.

g) Se han incorporado técnicas de comunicación que promuevan en el personal de almacén su integración y trabajo en equipo.

\subsection{Metodología}

Este REA contiene la formación para desempeñar las funciones de gestionar y coordinar las operaciones del almacén, optimizando los recursos disponibles.

La función de gestionar y coordinar las operaciones del almacén incluye aspectos como:

- La determinación de necesidades de recursos humanos y técnicos.

- La selección de equipos, medios y herramientas de almacén.

- Realizar planos de distribución interna de mercancías según métodos y técnicas de almacenamiento.

- El cálculo de coefícientes de utilización e índices de capacidad.

- Realizar y seguir protocolos de almacenaje.

- Realizar presupuestos de almacenaje de mercancías.

- Llevar a cabo la programación de mantenimiento del almacén.

- Relación con el equipo de trabajo.

\section{Proceso de trabajo}

\subsection{Elaboración de materiales}

Para el inicio del proceso de trabajo, se parte de la elaboración del mapa mental del REA: 
Tabla 2. Mapa mental del REA

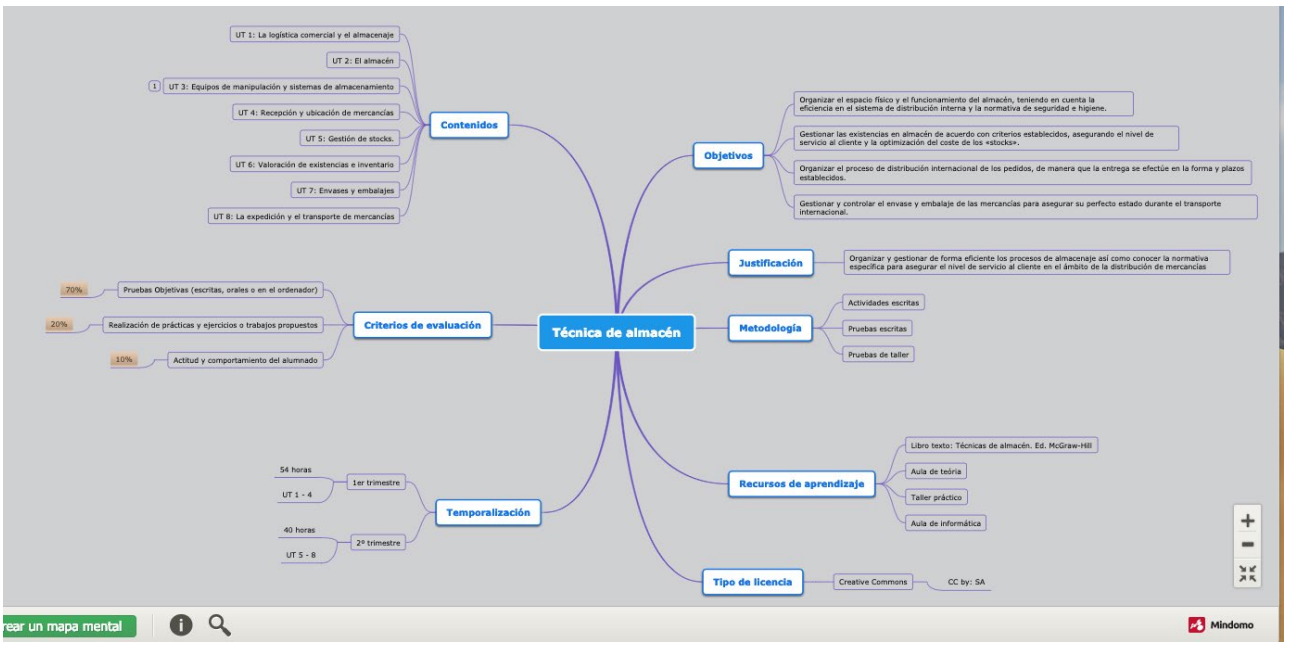

Fuente: Elaboración propia

https://www.mindomo.com/es/mindmap/tecnica-de-almacenaje-094a42d1ceab4c489708f7511153acd7

\subsection{Herramientas}

Para la creación de contenidos que posteriormente serán incorporados a Procomún, se ha utilizado la herramienta de autor eXelearning. Se trata, por tanto, de una herramienta de autor fácil de usar y con código abierto que permite que los materiales educativos se adapten a las necesidades de los profesores y los alumnos.

Seguidamente, se compartieron los recursos en el repositorio de recursos digitales educativos abiertos (REA) del Ministerio y las Comunidades Autónomas, Procomún.

El Espacio Procomún Educativo de EducaLab es un servicio del Ministerio de Educación, Cultura y Deporte que se enmarca en una de las líneas de trabajo del Plan de Cultura Digital en la Escuela.

\subsection{Temporalización}

Para los contenidos del REA han sido distribuidos en 4 Unidades de trabajo, traducidas en 54 horas totals en un trimester, lo que supone ( $5 \mathrm{~h} / \mathrm{semana}$ ).

Tabla 3. Temporalización del REA

\begin{tabular}{ll}
\hline UT 1: & La logística comercial y el almacenaje \\
\hline UT 2: & El almacén \\
UT 3: & Equipos de manipulación y sistemas de almacenamiento \\
UT 4: & Recepción y ubicación de mercancías \\
\hline
\end{tabular}

Fuente: Elaboración propia 


\section{Conclusiones}

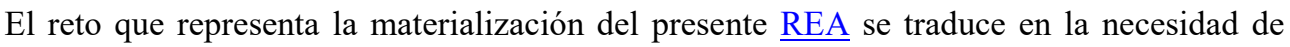
analizar y observar la oportunidad de colaborar y compartir que proporcionan, facilitan el intercambio de conocimientos, desarrollo de capacidades y acceso universal al aprendizaje y enseñanza de calidad.

El análisis de las conclusiones llevado a cabo es el siguiente:

- Estructura y organización: se describen y cumplen los objetivos, contenidos, indicadores competenciales, secuencia de actividades y criterios de evaluación para el Ciclo Formativo para el cuál ha sido creado. Además se incluye un apartado muy interesante de actividades secuenciadas de forma clara, que incluyen la solución. Se trata de un recurso muy bien planteado y muy útil y efectivo.

- Aplicación en el aula: se trata de un recurso muy útil y efectivo no sólo para su uso en las aulas, sino además para el módulo semipresencial. Es decir, se trata de un recurso perfectamente adaptable a la enseñanza a distancia.

Los aspectos más relevantes que particularmente destacaría son: la cantidad de actividades con solución que dispone, pues supone una herramienta clave para el aprendizaje de los contenidos. Además de los test de autoevaluación finales que ayudan al alumno a afianzar los conocimientos.

- Elementos de diseño: efectivamente contiene elementos de diseño de refuerzo como gráficos, esquemas de la cadena logística además de ilustraciones que ayudan al entendimiento del contenido siendo apropiadas para el nivel educativo y el perfil del alumno. En todo caso, se cita la fuente de origen. Se ofrece bajo la licencia CC BY-SA.

De este modo, mediante la introducción de metodologías innovadoras basadas en un aprendizaje colaborativo, se pretende que los alumnos aprendan de una manera diferente. Los objetivos de este proyecto facilitan la integración de diferentes áreas del currículo de los ciclos de educación superior.

\section{Referencias}

Congreso mundial sobre los recursos educativos abiertos (REA) UNESCO, París, 20-22 de junio de 2012. Disponible en: $<$ http://www.unesco.org/new/fileadmin/MULTIMEDIA/HQ/CI/CI/pdf/Events/Spanish_Paris_OER_ Declaration.pdf> [Consulta: 10 de marzo de 2019].

EDUTEKA (2007). Recursos Educativos Abiertos (REA). Recuperado de http://www.eduteka.org/OER.php

Horizon Report. Resumen Informe Horizon 2015. Enseñanza Universitaria. Instituto de Tecnologías Educativas Departamento de Proyectos Europeos. Disponible en: <https://intef.es/wpcontent/uploads/2015/08/Resumen_Horizon_Universidad_2015_INTEF_agosto_2015.pdf>

[Consulta: 10 de marzo de 2019].

INTEF (2017). "Curso Creación de Recursos Educativos Abiertos para la Enseñanza" Instituto Nacional de Tecnologías educativas y de formación del profesorado (FORMACIÓN EN RED) (201712-20). 
JOHNSON, L., ADAMS BECKER, S., ESTRADA, V., FREEMAN, A. (2015). "NMC Horizon Report: 2015“ Higher Education Edition. Austin, Texas: The New Media Consortium.

MORENO, B. (2007). La dimensión europea de la Educación: Una investigación evaluativa en torno al programa eTwinning. Granada: Universidad de Granada.

PAZ-ALBO, J., LÓPEZ, I. (2017). Higher education perspectives on etwinning: the future of initial teacher training learning. 11th International Technology, Education and Development Conference. It will be published by IATED in the INTED2017 Proceedings, 6th-8th March 2017, Valencia, Spain.

Portal oficial PROCOMÚN Disponible en: $<$ http://procomun.educalab.es/es $>$ [Consulta: 10 de marzo de 2019]. 